\title{
THE RISKS OF DENTAL EXTRACTION DURING PENICILLIN TREATMENT
}

\author{
BY \\ LAWRENCE P. GARROD AND PAMELA M. WATERWORTH \\ From the Department of Bacteriology, St. Bartholomew's Hospital \\ Received June 20, 1961
}

It is widely believed that should dental extractions be indicated in a patient with bacterial endocarditis, the best time for the operation is during the treatment of this disease. Brock (1952) in discussing not merely the removal of suspicious teeth but total clearance with the object of guarding against recurrence, writes:- "Moreover this decision should be made the first week of therapy so that the teeth may be removed when the patient has adequate penicillin coverage." The purpose of the present paper is to show that this is the exact opposite of the truth.

It has long been recognized that treatment with penicillin, at least in large doses, suppresses the penicillin-sensitive flora of the mouth, including most of the streptococci. They are replaced by Gram-negative organisms (Neisseria and Hamophilus) and sometimes by coliform bacilli and Candida albicans (Long, 1947; Lipman et al., 1948). It is not so generally understood that the flora established during treatment also includes streptococci which are much more resistant to penicillin than those normally present. There is therefore a risk that, should a bacteriæmia be caused by dental extraction during penicillin treatment and an endocarditis result, it will be highly resistant to the usual treatment. In a patient already suffering from bacterial endocarditis, the implantation of circulating bacteria on the vegetations can presumably occur much more readily than on the much smaller and smoother surface of an uninfected valve. Hence reinfection with a highly resistant organism is a distinct and dangerous possibility. Case 1 (below) is believed to be an example of this.

The timing of penicillin "cover" for dental extractions in patients predisposed to endocarditis has also to be considered in the same light. How varied practice is in this connection may be judged from the fact that the recommended intervals between the first dose of penicillin and the extraction are given as two days, one day, and 30-60 minutes in different chapters of the same book (Welch, 1954). Some authors (e.g., Coffin, 1953) believe that a course of penicillin should be given before extraction with the object, so far as possible, of sterilizing the mouth and any foci of infection in it. Others regard the purpose of giving penicillin as the destruction only of such bacteria as actually enter the circulation, and therefore advise giving the first injection at an interval before extraction which will provide the highest blood level at that time. This interval is about two hours if procaine penicillin is used: if "soluble" (i.e. sodium or potassium) penicillin, "a more appropriate interval is five minutes" (Garrod, 1953). If, on the other hand, a course of several days' treatment precedes the extraction, a resistant flora will already have been established in the mouth, with the risk that should an endocarditis result, it will be exceedingly difficult to treat. Case 2 is an almost certain example of this disastrous occurrence.

\section{CASE RePorts}

Case 1. A man, aged 39, was admitted to Farnham Hospital under the care of Dr. J. W. Todd in December, 1959, suspected of having bacterial endocarditis involving the aortic valve. This was confirmed 
by a positive blood culture, the organism found being a Streptococcus viridans sensitive to penicillin, streptomycin and other antibiotics. On December 15 a course of penicillin, 2 mega units and streptomycin $1 \mathrm{~g}$. daily was started: the temperature fell to normal in three days and he became symptom-free. On January 7, all his teeth were removed with a view to preventing later recurrence. Treatment with penicillin and streptomycin was continued until January 11, when it was replaced by penicillin V $1 \mathrm{~g}$. orally, four times a day. Before the end of the month, by which time he had returned home, fever and sweats had recurred, and blood culture on February 1 yielded Streptococcus viridans resistant to penicillin and streptomycin, although sensitive as before to tetracycline, chloramphenicol and erythromycin and sensitive to novobiocin. Novobiocin, $500 \mathrm{mg}$. q.i.d. given four times a day from February 11-18 did not reduce his fever and blood culture on February 19 again yielded the same streptococcus. On the following day a course was started of penicillin 20 mega units and streptomycin 2 g. daily with probenecid 0.5 g. q.i.d. Fever abated slowly and the temperature remained almost normal after the first week of treatment.

On February 29 Dr. Todd wrote to one of us (L.P.G.) for advice about further treatment, and Dr. C. G. Thomas of the Group Laboratory, Farnham Hospital, kindly supplied a culture of the streptococcus from the patient's blood. The minimum inhibitory concentrations of 10 antibiotics were determined: all were within normal limits except penicillin $8 \mu \mathrm{g} . / \mathrm{ml}$. (13.3 units) and streptomycin $>512 \mu \mathrm{g} . / \mathrm{ml}$. Tests of combined bactericidal action by the method of Martin, Sureau and Chabbert (1952) showed various combinations to be totally bactericidal, but the noteworthy feature of the results was that erythromycin (of which the minimum inhibitory concentration was $0.25 \mu \mathrm{g}$. $/ \mathrm{ml}$.) was totally bactericidal both alone and in combination with every other antibiotic tested. It was evident from these results that erythromycin should replace streptomycin, and the patient was then treated with penicillin 20 mega units daily and erythromycin $250 \mathrm{mg}$. four times a day and made a complete recovery. He is still well and leading an active life over a year later.

This is a patient originally infected with a streptococcus fully sensitive to all antibiotics, who had multiple extractions during a course of treatment with penicillin and streptomycin, and relapsed within three weeks, the infection then being due to a streptococcus highly resistant to these two antibiotics. It is inconceivable that such resistance could have been acquired by the original organism. The relapse can only be explained as a reinfection from the mouth, where a doubly resistant population had been selected by 23 days' treatment with these two antibiotics.

Case 2. The salient features of this remarkable case were briefly referred to by Cates et al. (1957). A man, aged 37, with a clear history of rheumatic fever at the age of 20, was admitted to this hospital on January 15,1946 , with a history of fever, joint pains and ankle swelling for eight weeks. He had collapsed that morning and had a right hemiplegia and aphasia and signs of aortic regurgitation. Blood culture yielded a growth of a Streptococcus viridans three times more sensitive to penicillin than the Oxford staphylococcus (i.e. inhibited by about 0.01 unit per ml.). He was given 500,000 units of penicillin daily for 14 days and 250,000 for a further 11 days. His temperature, which reached a maximum of $103^{\circ} \mathrm{F}$. shortly after admission, fell to normal in three days, and he made a complete recovery.

The main interest of his case is bound up with his subsequent dental history, of which there are fortunately very accurate records. He suffered from dental sepsis which was considered to call for further hospital admissions and multiple extractions on three subsequent occasions, as follows.

October 30, 1946: Extraction of four teeth, three of which had apical abscesses, at $12.00 \mathrm{hr} ; 50,000$ units of penicillin given at 11.55 (i.e., 5 minutes beforehand). Treatment continued with 62,500 units 4 times daily for four days. No sequelæ.

July 27, 1949: Extraction of six teeth; 300,000 units procaine penicillin were given $1 \frac{1}{2}$ hours beforehand and repeated on each of two successive days. No sequelæ.

August 29, 1950: Penicillin administration begun at the rate of 500,000 units six-hourly and continued until September 5; five teeth extracted on August 31 and five on September 2. Penicillin had thus been administered in considerable doses for two days before the first of these extractions and five days before the second.

This patient was readmitted on October 20 , having been noticed to be pale and thin ten days before, and having had a fit seven days before; three days before when he had been seen in the Out-Patients' Department, his E.S.R. was $42 \mathrm{~mm}$. (having been $6 \mathrm{~mm}$. and $11 \mathrm{~mm}$. before and after extractions respectively during the previous admission) and blood culture was positive. This culture, made on October 17 (i.e. before admission) yielded 22 colonies of Streptococcus viridans per ml.: a further culture on October 24 yielded 14 colonies per $\mathrm{ml}$. This organism was alpha-hæmolytic, did not ferment mannite, and was not of Lancefield's Group D; i.e., it was not Str. faecalis. It was inhibited by four but not by two units per ml. of penicillin: it was thus 
400 times more resistant than the organism causing the original infection nearly five years earlier. It was also sensitive to $3 \mu \mathrm{g}$. $/ \mathrm{ml}$. of streptomycin.

The patient was treated with 2.5 mega units of penicillin three-hourly and $1 \mathrm{~g}$. of streptomycin six-hourly for six weeks, the latter adding total loss of labyrinthine function to his already serious disabilities. He recovered completely from the infection, and survived until October 28,1956 , when he was reported to have died after the rapid onset of further paralysis proceeding to coma, believed by his doctor to be caused by a further cerebral embolus.

There can be no reasonable doubt that the second attack of endocarditis in this patient resulted from the multiple extractions carried out a little over a month before the onset, and that the penicillin given failed to protect him because it was given for much too long beforehand. The interval was such that a resistant mouth flora must have been established, and circulating penicillin failed to destroy the highly resistant streptococcus which gained access to the circulation. The fact that multiple extractions had been performed on two previous occasions with no ill effects, under penicillin cover started at appropriate short intervals before the operation, provides further confirmation of this belief.

\section{Observations on the Penicillin Sensitivity of Salivary Streptococci During Penicillin TREATMENT}

It seemed advisable to obtain more exact and extensive information than has existed hitherto about the effect of penicillin treatment on the susceptibility of streptococci in the mouth. Cultures made in this laboratory from the mouths of several patients in the past, in whom the advisability of dental extraction was being discussed, have always yielded resistant streptococci during a course of penicillin, but the degree of resistance was not accurately measured. The following observations have recently been made in order to provide this information.

Methods. The subjects investigated, comprising 16 normal controls, all laboratory staff, and 31 patients undergoing treatment with penicillin, were instructed to expectorate saliva into a sterile Petri dish. A very small inoculum of this was transferred with a $1 \mathrm{~mm}$. platinum loop to a blood agar plate and distributed uniformly with a glass rod spreader. A hole $8 \mathrm{~mm}$. in diameter previously cut with a sterile cork-borer in the centre of the plate was then filled with penicillin solution, 10 units per ml.

After overnight incubation, colonies which appeared to be those of streptococci were streaked on a second blood agar plate. These were taken from an area remote from the zone of penicillin inhibition, if any. A single colony of each different colonial type was chosen, the differences being in their action on blood, whether alpha-, beta, or non-hæmolytic, and in other characters such as size, degree of roughness, opacity, etc. From one to four subcultures, most usually two or three, were made from each primary culture, according to the variety of colonial morphology observed. These were examined microscopically to verify that they were streptococci, and then transferred to tubes of blood broth.

This culture was used for an accurate quantitative test of penicillin sensitivity. Plates were prepared containing 3 per cent of horse blood lysed with saponin (to permit inspection by transmitted light) and concentrations of penicillin (potassium benzyl penicillin) differing two-fold and based on 1 unit per ml. (Report, 1961). Divided areas on these plates were inoculated with a $2 \mathrm{~mm}$. loopful of an approximately 1 in 500 dilution of the blood broth cultures of the streptococci. These plates were read for the presence or absence of growth after 24 hours' incubation.

\section{RESULTS}

Typical examples of primary cultures from control and treated subjects are illustrated. Fig. 1 is that of a control: there are no colonies of streptococci within about $1 \mathrm{~cm}$. of the penicillin hole, the few dark colonies in this zone being those of a Neisseria. External to this zone are numerous colonies of streptococci, causing a diffuse change in the blood in the medium, mainly alpha-hæmolysis. Fig. 2 is a culture from a treated patient: the growth, which consists largely of streptococci, is unaffected by penicillin, there being no zone of inhibition whatever. 


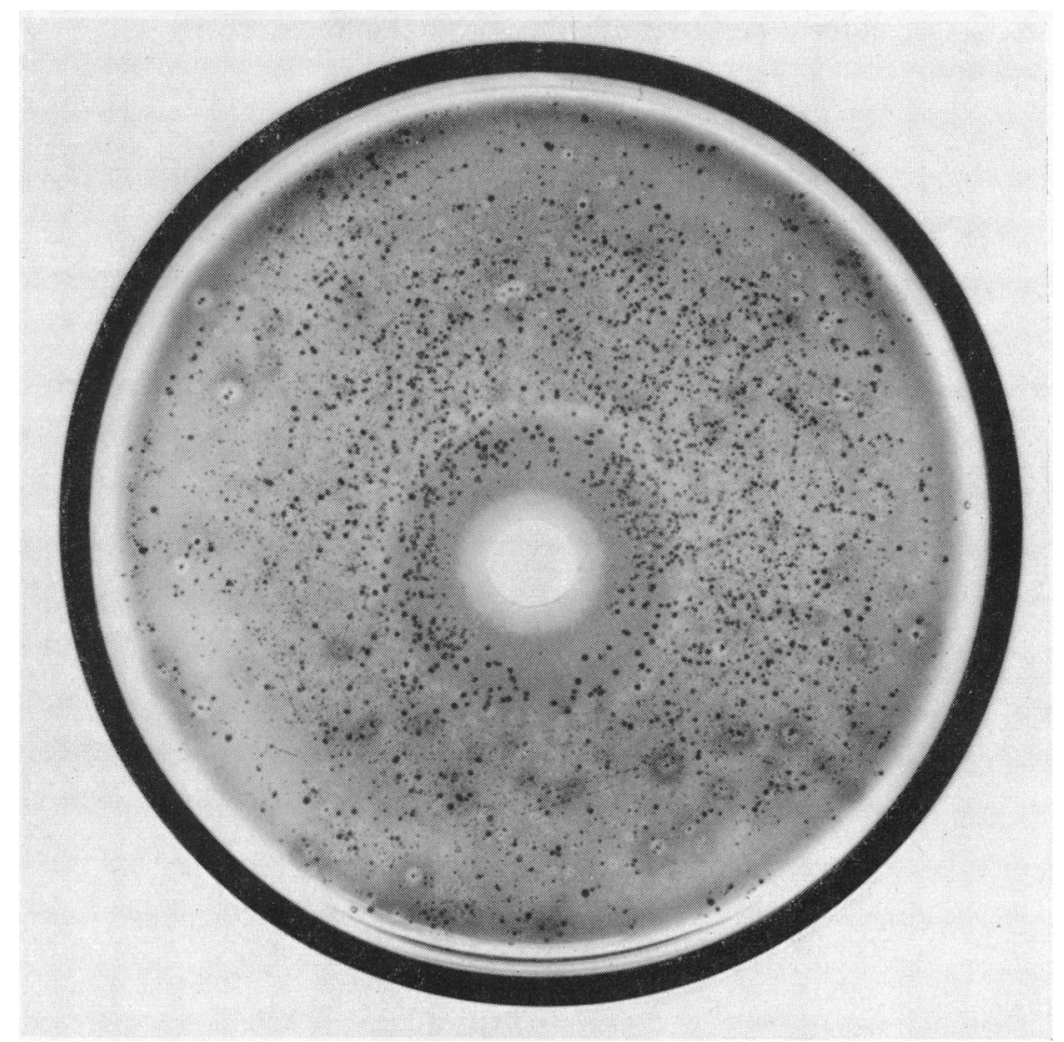

FIG. 1.-Photograph by transmitted light of blood agar plate culture of normal saliva. The hole in the centre contained penicillin solution, $10 \mathrm{units} / \mathrm{ml}$. The dark colonies in the inner zone of growth are those of a Neisseria. The nearest colonies of streptococci, recognizable by the hæmolysis which they produce, are $1 \mathrm{~cm}$. from the hole, indicating that they are of approximately normal sensitivity to penicillin.

The results of quantitative tests of sensitivity to penicillin are given in Table I. Of 40 strains of streptococci isolated from controls all but one were inhibited by $0 \cdot 25$ units per $\mathrm{ml}$. or less and mostly by 0.06 or 0.03 units per $\mathrm{ml}$. Of 74 strains from 31 patients undergoing treatment the great majority were inhibited only by concentrations varying from 2 to 32 units per $\mathrm{ml}$. All these were tested for mannite fermentation with negative results: therefore they were not Str. facalis.

\section{TABLE I}

Numbers of Strains of Salivary Streptococci from Control and Treated Subjects Inhibited by Different Concentrations of Penicillin

\begin{tabular}{|c|c|c|c|c|c|c|c|c|c|c|c|c|c|c|c|}
\hline & \multirow{2}{*}{$\begin{array}{c}\text { Number } \\
\text { of } \\
\text { subjects }\end{array}$} & \multirow{2}{*}{$\begin{array}{c}\text { Number } \\
\text { of } \\
\text { strains }\end{array}$} & \multicolumn{13}{|c|}{ Minimum inhibitory concentration (units per ml.)* } \\
\hline & & & -6 & -5 & -4 & -3 & -2 & -1 & 0 & 1 & 2 & 3 & 4 & 5 & $>5$ \\
\hline $\begin{array}{l}\text { Controls } \\
\text { treated }\end{array}$ & $\begin{array}{l}16 \\
31\end{array}$ & $\begin{array}{l}40 \\
74\end{array}$ & $\underbrace{5}$ & $\begin{array}{l}17 \\
-\end{array}$ & 11 & $\begin{array}{r}3 \\
-\end{array}$ & $\begin{array}{l}3 \\
5 \dagger\end{array}$ & $\overline{3}$ & $\begin{array}{l}1 \\
3\end{array}$ & 21 & 17 & 8 & 6 & 8 & 3 \\
\hline
\end{tabular}

* O denotes 1 unit per ml. Other figures are the $\log _{.2}$ of the difference from this-that is, 1, 2, 3, 4, denote 2, 4, 8, 16 units per ml. etc., $-1,-2,-3,-4$, denote $0 \cdot 5,0 \cdot 25,0 \cdot 12,0 \cdot 06$, etc.

$\dagger$ Including 3 strains for which no end point was obtained (i.e. possibly sensitive to a lower concentration). 


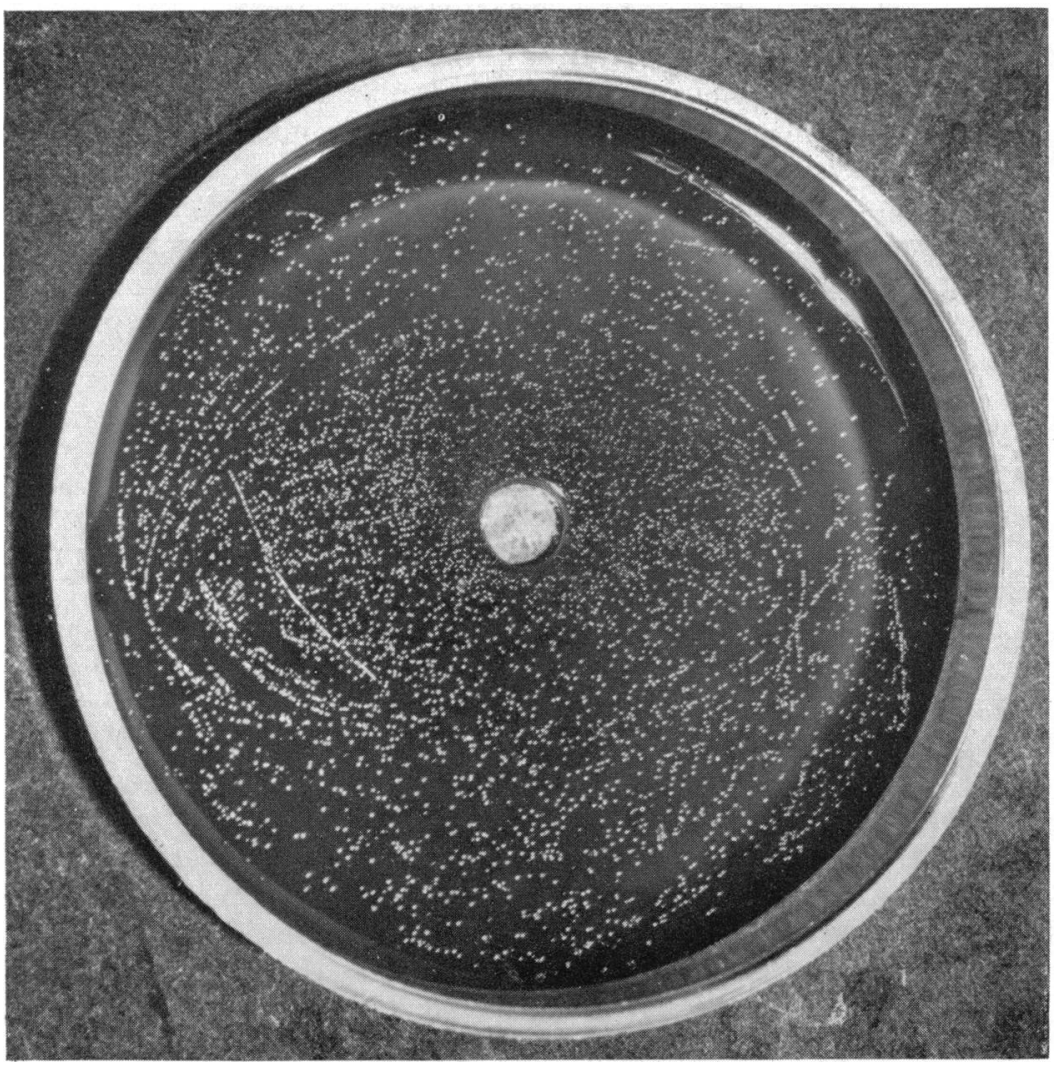

FIG. 2.-Culture of the saliva of a patient under treatment with penicillin, with a hole containing penicillin solution as in Fig. 1. The majority of the colonies are of streptococci, but there is no zone of inhibition, all the organisms being abnormally resistant. (Photographs taken by reflected light.)

To understand the full significance of these findings it is necessary to consider the form of penicillin treatment being given. All but four of the patients were being treated with only small oral doses of penicillin, in most cases for the prophylaxis of rheumatic fever: this group consisted of 26 children of ages ranging from 4 to 16 years and one woman aged 32 . The usual doses were 125 or $250 \mathrm{mg}$. penicillin V, two or three times a day or 200,000 units benzyl penicillin twice daily; the duration of treatment varied from a minimum of 10 days to many months. The remaining four patients had been receiving injections of penicillin for at least seven days, two having 1 mega unit twice daily, one the same dose four times a day and one methicillin $1 \mathrm{~g}$. four times a day (this patient's two strains of streptococci were inhibited by 4 and 8 units per ml.). This group is too small for comparison with the remainder, but so far as the eight strains isolated from them afford any indication, they appear to possess resistance of the same order as that of strains from patients receiving oral treatment.

A few observations have been made on what happens to the flora of the mouth when treatment is stopped. In five patients specimens were examined daily except on Sundays for 5, 5, 10, 10, and 17 days and in two patients also 41 and 42 days after the end of the course. These cultures were assessed only by the effect of the penicillin diffusing from the hole in the plate, no titrations being carried out. Penicillin-sensitive streptococci usually appeared after two days and increased in numbers, but some resistant colonies persisted, and in no culture had they entirely disappeared. That they must do so 
eventually is almost certain: several control subjects, in none of whom were resistant strains detected, were known to have received penicillin treatment in the past.

A small experiment has also been carried out to determine how soon after the start of treatment resistant streptococci appear in numbers in the mouth. Two normal adults were given $250 \mathrm{mg}$. penicillin $\mathrm{V}$ four times a day. After one day of treatment almost all streptococci had disappeared in both subjects, the culture from saliva consisting almost entirely of a Neisseria in one subject and a Neisseria and a Hamophilus in the other. After two days large numbers of colonies of resistant streptococci, growing right up to the edge of the penicillin hole, appeared in one subject, and smaller numbers of similar streptococci in the other. It therefore seems that a potentially dangerous resistant flora can be established within 48 hours.

\section{Discussion}

There is no record of any previous observation quite on the present lines, and certainly none of any comparable results. Lipman et al. (1948) cultivated throat swabs from 10 patients before, during, and after long courses of penicillin treatment, and observed the disappearance of pneumococci and Group A streptococci. They examined also the penicillin sensitivity of non-hæmolytic (including viridans) streptococci, but the records for this organism are complete in only three cases, and in only three does it appear that a more resistant strain was cultivated during than before treatment. Meads et al. (1957) gave $0 \cdot 2 \mathrm{~g}$. penicillin three times a day for " 4 or 5 days" to five subjects, cultivated throat swabs before, during, and after treatment, and found a "slight but significant increase" in resistance to penicillin in alpha-hæmolytic streptococci. The only observations on the mouth, as distinct from the throat, are those of Welch et al. (1952), but these concern children who had been using a tooth powder containing 500 units of penicillin per g. Gum swabs were taken from 185 children who had used this three times a day for three years and from 178 who had not, and numerous colonies of streptococci from each plate were subcultured and tested quantitatively for penicillin sensitivity. This was found to vary widely in both treated and control subjects, but the proportion of streptococci inhibited by concentrations in the range $0 \cdot 5-10$ units $/ \mathrm{ml}$. was higher in the treated.

The present results show a clear-cut difference between the mouth streptococci of normal subjects and of those undergoing systemic penicillin treatment. Presumably this is the effect of the presence of penicillin in the saliva. Long (1947) believed that penicillin is excreted in the saliva only when the dose given is 500,000 units a day or more, but Bender et al. (1953) found concentrations of up to 0.023 units $/ \mathrm{ml}$. in the saliva of each of 10 subjects one hour after an injection of 100,000 units of sodium and 300,000 of procaine penicillin, and it seems unlikely that there is any threshold for its excretion. The appearance of resistant strains could be the result of either selection or mutation, and no attempt has been made to determine which mechanism is operative, but selection is much the more probable. Penicillin is an antibiotic to which bacteria acquire resistance, whether by mutation or otherwise, only with difficulty and usually to a small degree. In favour of the selection hypothesis is the great variety of the mouth streptococci, and the fact that some authors have demonstrated the presence of highly resistant strains in the normal mouth. Among the findings of Welch et al. (1952) already referred to was that $4 \cdot 3$ per cent of streptococci from control (untreated normal) mouths were resistant to $1 \mathrm{unit} / \mathrm{ml}$ : this is a higher proportion than would be expected. These resistant organisms evidently form a small minority of the population of the normal mouth, but in the presence of penicillin they are enabled to multiply and replace the sensitive majority which have been suppressed.

If the thesis put forward here be accepted, it has applications to both the prevention and the treatment of subacute bacterial endocarditis. It is assumed here that penicillin "cover" for dental extraction is of benefit in subjects predisposed to bacterial endocarditis, although statistical proof of this does not exist, and some examples of failure have been recorded. Of those quoted by Glaser et al. (1948), two may have been due to inadequate dosage and one is of particular interest in connection with the present argument because the cover in this case was long-term penicillin admini- 
stration for the prevention of rheumatic fever. These authors' own experiments consisted of making blood cultures 2-5 minutes after extractions in 40 patients given penicillin cover and 40 controls: the percentages positive were 42.5 and 67.5 respectively. This apparently disappointing result is less significant than it seems, if account is taken of the rate of bactericidal action of penicillin. This antibiotic requires about 2 hours to cause a substantial mortality in a bacterial population and 4 hours or more to exterminate it. These authors used a medium containing penicillinase, and there was therefore not enough time for penicillin in the blood to kill the bacteria which had only just entered the circulation. Viewed in this way, the object of penicillin cover for extraction should be to maintain a bactericidal concentration of penicillin in the blood from the time of extraction for, say, 6-8 hours or possibly longer as a full precautionary measure. The process of destroying some of the liberated bacteria will be begun while they are still circulating and continued in any lodgment which they may find within the circulation, such as the surface of a damaged heart valve. This could be achieved by administering, say, 100,000 units of sodium or potassium penicillin and 300,000 units of procaine penicillin immediately before the extraction. A repetition of this dose at 12-hour intervals until 3 or 4 doses have been given would perhaps be a wise precaution, although the issue will probably have been settled by the first dose.

If a patient is already being given penicillin, as for the prevention of rheumatic fever, and dental extractions are necessary, what cover should then be given? In view of the present findings, even much larger doses of penicillin might be inadequate. The choice of another antibiotic is limited if the proposition be accepted that a fairly rapid bactericidal action is necessary. One possibility is vancomycin; this is strongly bactericidal and resistance to it in normally sensitive bacteria, including streptococci, is unknown. A single intravenous dose of $1 \mathrm{~g}$. should provide adequate cover, and has been used on several occasions in such circumstances in this hospital.

Dental treatment in established bacterial endocarditis is the final problem. It seems appropriate here to make a plea for an immediate dental overhaul as soon as the diagnosis is established or even seriously suspected. Since it is universally acknowledged that the source of infection in this disease is usually the mouth, should not the eradication of any focus there be the first step in treatment? It should usually be possible to X-ray the teeth and even to extract any with signs of apical infection while the diagnosis is being established by blood culture, or at least very shortly after this. According to the present findings, there is a possible risk of re-infection with a more resistant streptococcus if extractions are done more than one day after the beginning of a course of penicillin treatment. If they are deemed necessary later during the course or shortly after it, cover with a different antibiotic may be advisable, and vancomycin should be a suitable choice for this purpose.

The magnitude of the risk against which this policy is designed to guard is impossible to estimate, and may be small. On the other hand, the fact that other cases illustrating it have not been recorded is most unlikely to mean that none have occurred. Unless a possible connection between dental extraction during treatment and subsequent relapse is recognized there is no reason why they should be. In any further observations on the subject it is important that the sensitivity to penicillin of streptococci, if cultivated from the blood during a relapse, should be re-tested by an accurate multiple dilution method. Failure to re-test or reliance on the much less dependable disc method may conceal the fact that reinfection has occurred with a more resistant strain.

\section{SUMMARY}

During treatment with penicillin, even in small doses, the normal streptococci in the mouth are suppressed and replaced by penicillin-resistant strains. Almost all of 74 strains cultivated from the saliva of 31 patients during treatment were inhibited only by concentration of 1-32 units per ml. whereas the great majority of 40 strains from 16 untreated controls were inhibited by 0.25 units per $\mathrm{ml}$. or less.

Two case histories are recorded, one of a patient who suffered a second attack of bacterial endocarditis due to a highly resistant streptococcus following dental extractions carried out several days 
after prophylactic administration of penicillin had been started, the other of a patient who relapsed with infection due to a penicillin- and streptomycin-resistant streptococcus after dental extractions carried out during a course of treatment with these two antibiotics.

Penicillin "cover" for dental extractions should be begun immediately before the operation. Necessary dental treatment for a patient with bacterial endocarditis should if possible be carried out before penicillin treatment is begun. Should extractions be indicated during this treatment, they may be covered with a different antibiotic.

We are indebted to Professor R. V. Christie, Dr. G. W. Hayward, and Dr. J. W. Todd for permission to refer to their cases, and to other colleagues of the staff of this hospital and Professor E. G. L. Bywaters of the Canadian Red Cross Memorial Hospital, Taplow, for enabling us to obtain specimens of saliva from their patients.

\section{REFERENCES}

Bender, I. B., Pressman, R. S., and Tashman, Sylvia G. (1953). J. dent. Res., 32, 78.

Brock, H. J. (1952). N.Y. St. J. Med., 52, 607.

Cates, J. E., Christie, R. V., and Garrod, L. P. (1951). Brit. med. J., 1, 653.

Coffin, F. R. (1953). Brit. med. J., 2, 571.

Garrod, L. P. (1953). Brit. med. J., 2, 727.

Glaser, R. J., Danker, A., Mathes, S. B., and Harford, C. G. (1948). Amer. J. Med., 4, 55.

Lipman, Miriam, O., Coss, J. A., and Boots, R. H. (1948). Amer. J. Med., 4, 702.

Long, D. A. (1947). Brit. med. J., 2, 819.

Martin, R., Sureau, B., and Chabbert, Y. (1952). Bull. Soc. Méd., Paris, 68, 1192.

Meads, M., Rowe, W. P., and Haslam, Nancy, M. (1955). Arch. intern. Med., 87, 533.

Report (1961). Standardization of Methods for conducting Microbic Sensitivity Tests. World Hlth. Org. Technical Rept. Series No. 210, Geneva.

Welch, H. (1954). Principles and Practice of Antibiotic Therapy. New York, Medical Encyclopedia Inc.

-, Randall, W. A., Putman, L. E., and Hendricks, F. D. (1952). Antib. and Chemother., 2, 249. 